
Cahiers
Recherche
Fur les Droits
Fondamentaux

Cahiers de la recherche sur les droits fondamentaux

$4 \mid 2005$

Quel avenir pour la laïcité cent ans après la loi de 1905 ?

\title{
Interview de M. le Professeur Jean Baubérot
}

Directeur d'études à l'École pratique des hautes études, titulaire de la chaire « Histoire et sociologie de la laïcité »

\section{Gilles Armand}

\section{OpenEdition}

\section{Journals}

Édition électronique

URL : https://journals.openedition.org/crdf/7243

DOI : $10.4000 /$ crdf.7243

ISSN : 2264-1246

Éditeur

Presses universitaires de Caen

Édition imprimée

Date de publication : 1 décembre 2005

Pagination : 11-20

ISBN : 2-84133-250-0

ISSN : 1634-8842

Référence électronique

Gilles Armand, «Interview de M. le Professeur Jean Baubérot ». Cahiers de la recherche sur les droits fondamentaux [En ligne], 4 | 2005, mis en ligne le 15 décembre 2020, consulté le 19 novembre 2022. URL : http://journals.openedition.org/crdf/7243 ; DOI : https://doi.org/10.4000/crdf.7243 


\title{
Interview de M. le Professeur Jean Baubérot Directeur d'études à l'École pratique des hautes études Titulaire de la chaire "Histoire et sociologie de la laïcité "
}

\author{
Gilles ARMAND \\ Maître de conférences en droit public - Université de Caen Basse-Normandie \\ Directeur adjoint du CRDFED \\ Rédacteur en chef des CRDF
}

G.A.: En premier lieu, pourriez-vous rappeler, s'il vous plaît, l'apport historique de la loi de 1905 en ce qui concerne le rapport entre les Églises et l'État en France? Et, j'ajouterai: peut-on considérer que cette loi a constitué un tournant ou simplement la poursuite d'un mouvement de laïcisation enclenché depuis la Révolution de 1789?

J.B.: Je vais commencer par la fin et je vais remonter au début de votre question. Effectivement, la loi était présentée à l'époque comme parachevant l'œuvre de laïcisation réalisée par la Révolution française. Cette façon de présenter la loi a continué au XIX ${ }^{\mathrm{e}}$ siècle. Notamment Jaurès était un peu la personne qui a présenté la loi de cette manière avec beaucoup de verve et beaucoup d'allant. Ce n'est pas complètement faux. En même temps, vous avez tout à fait raison de parler d'un tournant. On peut dire que d'une autre manière la loi rompt sur certains points avec la perspective de la Révolution française et même avec l'anticléricalisme d'État qui s'était manifesté à partir de la République des républicains. Pourquoi ? Parce que finalement l'anticléricalisme a été particulièrement net dans la période 1899-1904, c'est-à-dire dans la période du processus qui a donné lieu à la loi sur les associations et avec le statut hors du droit commun réservé aux congrégations. Puis, dès 1904 c'est la loi interdisant aux congrégations d'enseigner. Dans ces années-là, on peut dire qu'il y a une alliance entre l'État républicain et la libre pensée au sens large: pas seulement entre l'État et les associations de libre pensée mais aussi entre l'État et tous ceux qui se réclamaient de la libre pensée. Quand il y a eu la Fête de la libre pensée en 1903, il y avait les Comités républicains, il y avait le Parti radical socialiste, le Parti socialiste, qui ont défilé, il y avait des représentants de la loge maçonnique, etc. Enfin, toute cette large mouvance libre penseuse était manifestement alliée à la République pendant ces années-là. La loi de 1905 c'est explicitement la loi de séparation des Églises et de l'État et il n'est pas question de nier cet aspect essentiel. Mais c'est aussi, Aristide Briand le dit, dès le 10 avril, au début de la discussion de la loi, la séparation implicite de la République et de la libre pensée. Aristide Briand dit très clairement qu'il faut que la loi permette à l'Église de se défendre de la libre pensée et que la libre pensée n'a pas à compter sur le pouvoir de l'État: elle doit compter uniquement sur sa propagande interne pour vivre et pour convaincre.

Donc, il y a ce premier aspect qui est déjà un tournant. Il y a un second aspect qui est un tournant : c'est le fait que sous la Révolution, je parle de la période libérale de la Révolution, je ne parle justement pas de la terreur politique ou religieuse, mais sous la période libérale de la Révolution qui à conduit à la Constitution de 1791, la liberté de culte est comprise comme un prolongement de la liberté individuelle. La Constitution de 1791 dit que tout citoyen est libre de pratiquer le culte auquel il est attaché. Donc, cette liberté collective est un prolongement de la liberté individuelle. Et c'est la même problématique qu'on retrouve dans la loi sur les associations de 1901 où les citoyens ont la liberté de s'associer librement pour rechercher un but commun. Et dans la pensée de Pierre Waldeck-Rousseau, une fois que ce but a été atteint l'association n'avait plus de raison d'être (néanmoins, la loi ne dit pas qu'une fois l'objectif atteint, l'association doit se dissoudre). Donc, on est vraiment dans la perspective où la liberté collective est un prolongement de la liberté individuelle. Et c'est d'ailleurs pour cela que les congrégations sont mises à part parce qu'elles sont des groupes qui existent en dehors de l'individu auxquelles l'individu s'agrège. Même des gens qui étaient des libéraux, qui n'étaient pas des gens sectaires 
comme Ferdinand Buisson qui après tout était le président de la commission, qui était le préparateur et qui était adjoint de Jules Ferry - une grande figure emblématique de la laïcité. Pendant les discussions sur la loi, Ferdinand Buisson dit bien que la loi ne doit plus connaître les Églises puisqu'elle se sépare d'elles; elle ne doit connaître que les citoyens et ces citoyens, quand ils sont catholiques, ont le droit de se réunir pour célébrer le culte catholique, quand ils sont protestants..., etc. Et ils ont le droit de le faire à leur manière, comme ils le veulent et cela ne regarde plus l'État. Alors que la loi, avec l'article 4 qui a été au cœur de discussions et qui a créé un conflit interne entre les républicains, dit que les associations devront se conformer aux règles générales d'exercice du culte dont elles se proposent d'assurer l'exercice. Cela veut dire que la loi tient compte des constitutions que se donnent les Églises. Le grand problème est de recevoir ce grand parc immobilier et culturel en sachant que c'est à la fois un problème matériel mais que c'est aussi un problème de légitimité. Pour la grande masse de Français qui se réclament du catholicisme, c'est l'église du village, du bourg, du quartier qui est la référence. Ces églises n'étant remises qu’à ceux qui se conformeront aux règles générales d'exercice du culte dont elles se proposent d'assurer l'exercice. Cela veut dire que même si la majorité d'une paroisse veut prendre son autonomie par rapport à l'évêque, par rapport à Rome, etc. l'église ira à la minorité qui restera fidèle à l'évêque, à Rome, etc. Et Buisson tempête contre cela, et il n'est pas le seul: beaucoup de républicains, tout au cours des discussions sur la loi, tempêtent contre cela, en disant que cela rompt avec l'idée républicaine justement selon laquelle c'est l'individu qui prime et non pas le groupe intermédiaire. Et la réponse de Buisson n'est pas une réponse qui se base sur les principes républicains mêmes, sur cet universalisme très républicain mais qui se fonde sur la nécessité de faire une loi acceptable par l'Église catholique. Le leitmotiv de Buisson est repris par Jaurès, et l'idée de Jaurès est qu'il faut faire des lois « acceptables » par l'Église catholique. C'est à cause de cette préoccupation d' " acceptabilité » que je parle du pacte laïque. Cela ne veut pas dire qu'il y eût des négociations : Buisson n'a jamais parlé de telles négociations. Mais le souci premier était de faire des lois acceptables par l'Église catholique.

G.A. : La deuxième question qui se pose est de savoir si la loi de 1905 peut être résumée au principe de séparation des Églises et d'État? Finalement, le respect de la liberté religieuse, sous ses diverses formes, est-il absent du texte?

J.B.: Tant qu'il y a séparation des Églises et de l'État, le titre de la loi est très explicite, c'est-à-dire qu'à mon sens, l’État, lui, était déjà séparé des Églises, de manière stable depuis Napoléon. D’ailleurs il est bien dit sous Napoléon : «Désormais l'Église est dans l'État et l'État n'est plus dans l'Église. » Et l'État s'est laïcisé, le Code civil des Français de 1804 ne contient aucune mention des normes religieuses, ne fait absolument pas référence à la religion. Mais, en revanche, les Églises ont recours à l'aide d'État. C'est double: c'est-à-dire qu'effectivement, elles ont certains privilèges - je dirais de semi-officialité, le clergé payé par l'État, etc. Ainsi, l'État protège les Églises mais aussi l'État les contrôle et les contrôle d'une manière assez étroite. Un exemple: un évêque ne peut pas quitter son diocèse sans l'autorisation du pouvoir civil. Et le fait que le Vatican demande à Monseigneur Le Nordez et à Monseigneur Gay de le faire en 1904 est une des causes qui est considérée comme une rupture du Concordat par le Saint Siège. Les Assemblées d'évêques ne sont pas vraiment interdites mais elles n'auront lieu qu'avec l'autorisation du gouvernement. Au XIX ${ }^{\mathrm{e}}$ siècle aucun gouvernement, même ceux considérés comme les plus cléricaux, ceux de la Restauration et du début du Second Empire, n'a jamais donné une autorisation à des évêques de se réunir. Pour les protestants, ils en ont eu une fois pour un synode en 1972 alors que le synode est quelque chose d'essentiel dans le gouvernement des églises réformées. Quand les évêques font une pétition ou une lettre collective, il y a une procédure pour abus engagée auprès du Conseil d'État parce que c'est la violation du Concordat. Cela est dit très explicitement durant la discussion sur la loi. La loi fait rentrer les Églises dans le droit commun, la loi donne des nouvelles libertés aux Églises et c'est pour cela d'ailleurs que certains députés, notamment dans le parti radical, étaient très réservés face à la tournure que prenaient les choses. Ils trouvaient que la loi était trop libérale étant donné ces libertés nouvelles. Ainsi, la séparation c'est la fin, effectivement, de certains privilèges, la fin de différences entre les cultes reconnus et les cultes non reconnus, mais l'idée de l'époque c'est plus de liberté. D’ailleurs, les évêques se réunissent tout de suite après la loi de séparation. Le pape nomme des évêques alors qu'avant ce n'était pas lui qui les nommait, c'était le pouvoir civil. Les évêques se réunissent et prennent des positions, ce qui n'était pas possible avant. Autrement dit, depuis le départ, l'Église catholique a utilisé les atouts et les libertés nouvelles que lui donnait la séparation. La liberté devient ainsi plus complète après la séparation. Évidemment, la jurisprudence a confirmé cette liberté dans la mesure où la crainte de certains catholiques, c'était que la loi soit libérale mais que cela soit un leurre et qu'ensuite l'application de la loi soit plus répressive. Or, ce n'était pas le cas. Au contraire, la jurisprudence a confirmé le libéralisme de la loi.

G. A. : Nous arrivons à la troisième question: à partir de ces éléments qui apparaissent dans la loi de 1905, c'est-à-dire séparation mais aussi liberté religieuse, aujourd'hui quelle définition peut-on donner de la lä̈cité? Et peut-on considérer que cette définition a évolué depuis 1905 ?

J.B.: Je crois qu'au niveau des principes la définition qui est finalement toujours valable est une définition que l'on peut tirer de Ferdinand Buisson. Il n'a pas explicitement, clairement dit ce que je vais vous dire, mais on en trouve des éléments dans l'article «Laïcité » du Dictionnaire de pédagogie écrit en 1883 , c'est-à-dire peu de temps après que le terme même de laïcité soit créé (puisque ses premières 
occurrences datent des années 70) - cet article est le premier grand texte théorique sur la laïcité. Ainsi, dans ce texte, il y a une sorte de triangle qui forme le principe laïque.

Le premier principe est un mouvement de prise de distance de l'État et de l'institution de la société civile: école, médecine, etc. par rapport à la religion. C'est d'ailleurs ce que Ferdinand Buisson appelle le long processus de siècles où peu à peu l'État se dégage de la tutelle de la religion et où les institutions peu à peu émergent et se développent d'une manière autonome. C'est, on peut le dire, le processus de laïcisation.

Mais le processus de laïcisation ne suffit pas à faire la laïcité et la preuve est que F. Buisson fait remonter la laïcisation à plusieurs siècles. Il dit que l'on ne peut parler de la laïcité qu'à partir de la Déclaration des droits de l'homme et du citoyen de 1789 parce qu'il y a là la proclamation de la liberté de conscience et on peut dire que sa conséquence est la liberté de culte.

Et puis, il y a aussi l'égalité de tous les citoyens devant la loi quelque soit leur appartenance religieuse ou non religieuse. Autrement dit, il y a aussi ces deux autres aspects : liberté de conscience et ses prolongements au niveau du culte et au niveau de la liberté de conviction et de religion et l'égalité devant la loi quelque soient les convictions et les religions de chacun. Ce triangle-là me semble toujours valable.

Ce qui maintenant est peut-être posé plus explicitement qu'il y a un siècle, c'est de se dire que l'égalité ce n'est pas tellement l'égalité d'un individu pris comme élément interchangeable, si je puis dire, de la société. C'est l'égalité des gens qui ont des appartenances et des références multiples tout en veillant à ce que le minoritaire ne soit pas désavantagé par rapport au majoritaire. Un exemple : le rythme de la société avec les jours fériés a fait que les gens qui sont chrétiens sont avantagés. Puisque le dimanche est le jour férié par excellence, puisque les principales fêtes chrétiennes sont chômées. Du coup qu'en est-il des gens qui appartiennent à d'autres confessions religieuses? Voici quelques points d'interrogation mais, comme vous le savez, la Commission Stasi avait proposé d'aller plus loin justement pour mieux réaliser l'égalité entre les citoyens. Les questions nouvelles qui se posent : quel est le degré de reconnaissance de la diversité qu'une société peut avoir tout en étant laïque, sans déroger au principe de la laïcité? Je pense qu'il y a une bonne méthode de travail qui d'ailleurs avait été comprise dans le rapport de la Commission Stasi puisqu'on en trouve référence à quatre reprises: c'est la notion d' " accommodement raisonnable». Une notion québécoise mais la Commission Stasi l'a faite sienne car elle l'emploie à quatre reprises. On peut dire d'ailleurs que l'article 4 de la loi de séparation est déjà un accommodement raisonnable. Accommodement donc parce qu'il faut lutter contre les discriminations indirectes. Il ne faut pas qu'une loi qui apparaît impartiale dans sa généralité favorise en fait implicitement certaines personnes par rapport à d'autres. Il faut que l'accommodement reste raisonnable, il ne faut pas que la diversité empêche l'unité, qu'elle rende impossible le lien social, qu'elle rende impossible le fonctionnement des institutions. Il faut arriver à faire ce genre d'accommodement réciproque où la société, les institutions, s'accommodent vis-à-vis des minorités mais où les minorités s'accommodent aussi de la nécessité du lien social.

G.A.: Puisque vous parlez de l'accommodement, est-ce que l'on peut considérer, encore aujourd'hui, que l'opposition classique, la séparation entre les sphères publique et privée, est encore pertinente pour déterminer les conditions d'exercice et les limites de la liberté religieuse? Finalement, peuton considérer que le pluralisme est cantonné à la sphère privée et demeure exclu de la sphère publique?

J.B.: Alors, je crois qu'il y a des modifications, sur ce plan, qui sont très fortes. Ce qu'il faut bien comprendre, c'est qu'à partir du moment où la religion est mise dans la sphère privée - et ce qu'il faut préciser, c'est un moment ou la sphère privée s'étend et la liberté dans la sphère privée est en train de s'étendre aussi - c'est le moment où il y a une démocratisation de la sphère privée. L'alphabétisation permet aux gens d'écrire des lettres sans contrôle et d'en recevoir, de les lire, de lire des ouvrages. L'école a une maîtrise de la sphère privée beaucoup plus grande : quand les lectures étaient des lectures publiques parce qu'il y avait une personne qui savait lire et les autres qui ne savaient pas lire, il n'y avait pas de sphère privée. De même, le métier était pratiquement imposé de père en fils. Seulement, à la fin du XIX ${ }^{\mathrm{e}}$ siècle, les gens commencent à choisir leurs métiers, à ne pas faire forcement le métier de leurs pères, etc.

Autrement dit, ce qu'on appelle le passage de la religion vers la sphère privée s'est fait à un moment où la sphère privée s'étend et où le fait que la religion ne soit plus socialement imposée mais soit une dimension de la liberté individuelle n'est pas finalement une mauvaise chose pour la religion puisque c'est un peu le mouvement de l'histoire : la diminution des obligations et l'augmentation des libertés individuelles. Mais en fait, ce n'est même pas stricto sensu la sphère privée : c'est le fait que l'institution religieuse est privatisée. Ce n'est plus un service public de religion classique. L'institution religieuse est privatisée au moment où par contre l'école est devenue une grande institution publique, où la médecine est devenue une grande institution publique.

Comme je le disais tout à l'heure à propos de la séparation, quand les évêques se mettent à pouvoir se réunir collectivement et à pouvoir parler sans qu'il y ait de procédure pour abus, cela veut dire que leur parole dans l'espace public est libre, peut s'exprimer librement, alors que sous le Concordat, ils étaient de la sphère publique et étatique avec les contraintes de la sphère publique et étatique. Tandis que là, ils sont dans la société civile, ils sont plus dans la société civile que dans une sphère privée étroitement entendue, ils peuvent s'exprimer en tant qu'éléments de la société civile dans l'espace public. Néanmoins, il faut bien comprendre ce qu'on entend par public et privé, car cela joue pour certains aspects, cela ne joue pas pour d'autres. 
Aujourd'hui, il y a une euphémisation des frontières entre le public et le privé. Le message social dominant, c'est le message publicitaire et je dirais le message médiatique. Il y a une certaine euphémisation entre le message publicitaire et le message médiatique. Quand vous prenez une publication comme Elle et que vous regardez rapidement les photos de Elle, vous ne savez plus ce qui est de la publicité et ce qui est de la photo de journal. Le message dominant est le message publicitaire et le message médiatique s'organise autour de ce message publicitaire. Or, ces moyens de communication de masse sont des moyens de communication qui sont dans la sphère privée. Alors que l'école et l'hôpital sont des lieux très localisés. La télévision, vous l'avez dans votre salle à manger, éventuellement dans votre chambre, le téléphone aussi vous l'avez dans votre appartement, Internet également. Les moyens de communication de masse abolissent la distinction sphère publique / sphère privée. Ceci à tous les niveaux: au niveau de l'instrument lui-même mais aussi au niveau de contenu. À voir le développement et le succès de toutes ces émissions comme par exemple Les confessions intimes ou les émissions de télé-réalité qui prétendent rendre public ce qui normalement appartient à la sphère privée. Quand vous avez une caméra qui vous filme $24 \mathrm{~h} / 24 \mathrm{~h}$... Par ailleurs, il faut bien voir que dans le temps il y avait une distinction relativement rigide entre sphère publique et sphère privée. Cela allait de pair avec le fait que la sphère privée était un peu une sphère de non-droit. Pendant des décennies, les affaires de pédophilie, les affaires d'inceste, les violences conjugales aussi, tout le monde savait que cela existait mais le droit n'allait pas regarder ce qui s'y passait. Maintenant, de nouvelles représentations des droits de l'homme font que ce n'est plus supportable. Et, justement, les moyens de communication de masse modernes ont aidé à ce niveaulà. Ils ont éveillé la conscience du public sur le fait que les délits et les violences qui se passent dans le privé sont autant punissables que ceux qui se passent dans le public. Là aussi il y a eu une euphémisation complète entre le public et le privé. Donc, aujourd'hui, je crois que les gens qui disent: «c'est la sphère privée, ce n'est pas la sphère publique » sont en complet décalage avec les mutations sociales et ils raisonnent d'une manière complètement rétrograde par rapport à ce que pense le Français moyen. Paradoxalement ce sont des philosophes qui vont vous dire cela.

Ce que je remarque, c'est que ces philosophes parlent beaucoup des Lumières, mais la pensée ne s'est pas arrêtée là. En plus, ce sont les Lumières françaises, c'est étroitement localisé. Je les entends très peu citer des idées européennes autres que françaises. Et puis on n'est plus au $\mathrm{XVIII}^{\mathrm{e}}$ siècle. La réflexion a évolué, les représentations ont évolué, il y a des débats internationaux actuellement, ne serait-ce qu'au niveau philosophique, auxquels je ne les entends pas beaucoup participer : ils ont quand même une pensée très rétrograde. En plus, quand ils parlent d'histoire, cela devient franchement comique car ils croient qu'il suffit de parler du passé pour être historien... Mais cela n'est pas vrai. Il y a toute une historiographie, il y a des études à partir des travaux d'archives. Les pratiques, les concepts, les notions propres aux historiens, ils ne les maîtrisent pas. Je ne leur reproche pas du tout de ne pas les maîtriser, ils sont philosophes. Il y a quand même une certaine naïveté du philosophe qui se croit «Monsieur ou Madame Je sais tout » et qui croit pouvoir parler de tout avec compétence. Non, ce n'est plus possible maintenant. Ils ont très souvent un savoir qui date énormément, et une conceptualité qui date et qui n'est plus du tout adaptée à la situation actuelle.

G.A.: Peut-on considérer que, dans son principe ou dans ses modalités d'application, la laïcité française est discriminatoire? Dans l'affirmative, pour quelles raisons, et quels remèdes pourraient être envisagés pour mettre un terme à ces discriminations?

J.B. : «Discriminatoire» serait un bien grand mot... Je crois quand même que la laïcité française, d'une manière générale, respecte un dispositif juridique assez important pour respecter les libertés et pour essayer d'articuler les différentes libertés entre elles. Ce qui n'est pas facile. Il y a, effectivement, pour moi deux points qui font problème, mais je ne les transforme pas en structure globale ou en structure d'ensemble. À ce niveau-là, on pourrait dire qu'aucun pays ne réalise une laïcité ni une liberté absolues. Ce qui est embêtant, ce que ces deux points, il faut qu'ils aillent ensemble, donc il y a une évolution un peu inquiétante de la laïcité dominante aujourd'hui en France qui se crispe pour un certain nombre de raisons et des peurs.

Le premier point, c'est la loi de 2001 qui se veut une loi contre les sectes et les groupements sectaires qui commettent des délits. Normalement, il y a des lois prévues pour cela et elles doivent fonctionner. En revanche, il est clair que cette loi de 2001 a créé une atmosphère de suspicion vis-à-vis de tout ce qui n'est pas considéré comme religieusement correct par des hommes qui ne savent pas forcement ce qu'est le religieux et qui n'ont surtout pas de culture du pluralisme religieux. Tous les ennuis qu'a la Fédération protestante de France, ainsi qu'un certain nombre d'Églises protestantes, viennent notamment de là. Si la Fédération protestante a demandé la modification de la loi de 1905, je crois qu'elle s'est trompée de cible. Ce n'est pas la loi de 1905 qui est responsable des problèmes concrets, réels, qu'elle éprouvait. C'est l'application qui en est faite par l'administration, dans le contexte de cette loi de 2001. Depuis 10 ans, il y a des problèmes dans le cadre d'un climat de suspicion contre tout ce qui ne serait pas religieusement bien estampillé. Le protestantisme avec son pluralisme interne est beaucoup plus difficile à estampiller religieusement que le catholicisme. Donc, il y a, effectivement, ce premier point. Ce que l'on peut dire aussi, c'est que le poids de l'administration en France, que l'arbitraire de l'administration par rapport au judiciaire, est un mal français qui contribue à créer des problèmes parfois sérieux.

Le second point, c'est la loi du 15 mars 2004 à laquelle je me suis personnellement opposé. Autant j'estime que le Conseil d'État avait tracé la juste limite entre la liberté 
d'expression, la liberté de conscience et les contraintes nécessaires, normales de l'institution scolaire. Il est évident que l'institution a ses propres contraintes, qu'elle a sa propre structure qu'il faut respecter et une certaine autorité institutionnelle qui doit être respectée pour qu'elle puisse fonctionner. Autant, je pense que prétendre que certains signes sont en eux-mêmes ostensibles, là, sur un point précis puisqu'il s'agit de se limiter à l'école publique, on a franchi un peu une ligne jaune. Cette loi s'est fait accompagner par des intolérances plus larges: une vielle dame qui a été refoulée car venant à une cérémonie où sa fille devait acquérir la nationalité française et elle portait un foulard, etc. Je suis amusé de voir que pendant la cérémonie officielle à laquelle assistait Jean-Pierre Raffarin pour la commémoration de la loi de 1905 à l'Académie des sciences morales et politiques, il y avait dans la salle trois jeunes femmes avec des foulards et qui les ont portés d'une manière tout à fait discrète... Je suis persuadé que la plupart des gens ne l'ont même pas vu, et la République n'a pas du tout été en danger, la République n’a pas du tout été menacée. Et justement, cette idéologie de la République menacée ressemble plus au temps du Combisme, plus qu'à la loi de séparation de 1905. Et effectivement, ce que l'on peut dire c'est que la laïcité française au niveau empirique, au niveau de sa réalisation historique, a toujours été tiraillée entre les périodes dites de combat qui se sont souvent accompagnées de certaines manifestations d'intolérance. Certaines: je ne voudrais pas exagérer. Il y a eu des problèmes très précis et, sans doute, de mauvaises réponses à ces problèmes. Et puis il y a eu des périodes beaucoup plus fastes au niveau de la liberté. Comme le moment de la rédaction de la Déclaration des droits de l'homme et du citoyen ou le moment de l'élaboration de la loi de 1905.

\section{G.A.: Vous apparaît-il opportun de renouveler l'expérience} en proposant de nouvelles lois, que certains estiment nécessaires, qui viendraient réglementer la liberté religieuse dans d'autres services publics?

J.B. : Je crois qu'il y a une croyance un peu sacralisée dans le pouvoir de la loi. Je pense que le recours à la loi doit évidement exister mais d'une manière pondérée et pas excessive. Cette tendance à légiférer de manière forte traduit plus des craintes qu'elle ne résout de véritables problèmes. Au demeurant, je me méfie aussi de cela puisque très souvent quand on fait des lois il y a d'autres raisons que la loi elle-même qui entrent en jeu. Par exemple, personnellement, je pense que dans la loi du 15 mars 2004, il y a eu, chez les législateurs, l'idée de calmer un certain nombre d'enseignants après la crise au niveau des retraites. Il y avait eu une grève très forte, très prolongée, qui avait laissé un souvenir amer à un certain nombre d'enseignants et on a cru leur faire plaisir et les calmer. Je ne suis pas sûr qu'on ait fait plaisir à tout le monde parce qu'il y a des gens qui avaient résolu, par des compromis raisonnables, le problème des foulards. Par exemple en autorisant les bandanas. On les a plutôt mis en difficulté par une loi qui n'était pas souple, plutôt que l'inverse. Donc, il faut faire atten- tion, parce que dans la loi de 2004, il y a eu des rivalités politiques qui ont joué, comme la rivalité entre Juppé et Sarkozy. Sarkozy était contre la loi donc Juppé était pour et il avait le vent en poupe à ce moment-là. Il y a eu aussi la volonté récurrente de Fabius, c'est-à-dire faire à peu près n'importe quoi pour apparaître plus à gauche que tout le monde. Quand François Hollande et Jean Glavany ont été auditionnés par la Commission Stasi, ils nous ont dit qu'ils ne pouvaient pas prendre des positions officielles parce que le parti socialiste ne s'était pas prononcé. Mais il n'y a qu'à relire le script de leurs propos. Leurs propos étaient très, très réservés face à cette loi. Laurent Fabius a poussé le parti socialiste à déposer un projet de loi interdisant tous les signes « visibles ", ce qui était à mon avis particulièrement stupide parce que je le mets au défi de me faire une liste de tous les signes religieux. Parler de tous les signes religieux visibles, cela veut dire que chaque proviseur doit avoir une liste exhaustive de signes religieux.

Donc, là, il y a vraiment des gens qui veulent faire des coups médiatiques, et qui ne réfléchissent pas sérieusement avant de faire des choses qui ont ensuite des conséquences qui les dépassent et qu'ils ne contrôlent absolument pas. Je me méfie actuellement de la manière dont nos législateurs font des lois. Je ne voudrais pas céder à un antiparlementarisme qui est facile mais parfois, malheureusement, les parlementaires donnent prix à cet antiparlementarisme. Le rapport qui avait été fait sur les sectes en 1984, tout le monde dit qu'il y avait des erreurs historiques énormes, que ce n'était pas un rapport qui manifestait une intelligence très brillante. Donc, il faut faire attention que les parlementaires ne se discréditent pas non seulement eux-mêmes, mais finalement, qu'ils ne discréditent pas le régime démocratique en ne se montrant pas d'une intelligence et d'une finesse suffisantes. Il y a parfois des dérapages pour des motifs de politique politicienne qui nuisent à la République.

G.A.: Que pensez vous du sort réservé en France à l'Islam? Considérez-vous à ce titre que la création du Conseil français du culte musulman (CFCM) constitue un progrès?

J.B.: Je dirais qu'il ne faut pas faire d'évangélisme. Il est normal qu'il y ait des tâtonnements face à l'Islam. On sait qu'il y a un passé conflictuel, des colonisations, on sait qu'il y a une hétérogénéité culturelle. On sait que la laïcité, que la loi de 1905 n'a pas été appliquée en Algérie, qu'on a parlé des Français musulmans. Personnellement, j'avais même vu une décision du Tribunal d'Alger, de 1903, qui parlait $d^{\prime}$ ' indigènes musulmans chrétiens ». Donc, il y a un passé très lourd. Et, comme vous le savez, il y a eu une mutation de l'immigration musulmane en 1975, d'une immigration d'hommes seuls qui avaient un projet de retour dans leur pays d'origine à une immigration des familles à un moment où on était à la fin des années glorieuses et où le chômage avait déjà démarré. D’ailleurs, il y a un lien entre cette crise socio-économique et la mutation de cette immigration à un moment où il y avait une déstabilisation des 
institutions par mai 68 au nom d'une utopie. Et cette utopie était en train de chuter, justement, avec la crise économique. On est passé de deux filières scolaires (une filière pour la bourgeoisie: petites classes de lycée, et une filière populaire : école primaire, école primaire supérieure, collège, etc.) à une école démocratique de masses rassemblant tout le monde. On a jamais pu arriver à bien maîtriser cette mutation qui était nécessaire, qui s'est faite tard et qui, puisqu'elle se faisait tardivement, s'est faite brutalement. Donc, il y a eu une sorte d'handicap. Un handicap qui a fait que les choses ne pouvaient pas se passer de manière idyllique et que c'est tout a fait logique qu'il y ait eu des tâtonnements. Ce n'est pas un discours qui cautionne ce handicap. Au contraire, il faut reprendre les choses en mains et il faut que l'Islam soit une des religions présentes dans l'espace français, dans la République. D’ailleurs, je rappelle que sous la III République, au moment des colonies, on parlait de la «France puissance musulmane». Il y a ce passé qui était conflictuel mais qui n'a pas été que conflictuel et on pourrait essayer de retrouver ce qui était positif. Comme, par exemple, la construction de la Grande Mosquée de Paris en 1925, où il y a eu des fonds de la ville, les fonds de l'État, etc. Il y a des choses que l'on pourrait revisiter pour voir que le passé est bien plus complexe que son souvenir. Ceci dit, à partir de là, il y a eu ce processus de création d'une instance représentative du culte musulman dont le but est d'appliquer à l'Islam la loi de 1905. Si on prend, par exemple, l'article 2 de la loi de séparation qui dit que la République ne reconnaît aucun culte, ce même article prévoit des aumôneries. Il n'y avait pas d'aumôniers musulmans faute de quelqu'un ou d'une instance avec laquelle la République puisse parler pour discuter de ce problème. J'estime que ce processus est globalement positif. Que l'on puisse adresser des critiques sur la manière dont cela a été fait, c'est fort possible. Ce qui est intéressant, c'est que les musulmans ont une représentation élue, alors que ce n'est pas le cas des catholiques et que ce n'est pas le cas de toutes les confessions. Pour les protestants, il s'agit des élus mais à plusieurs degrés. Pour les juifs cette représentation est élue mais à d'autres conditions. Mais, par exemple, le Conseil épiscopal français n'est pas élu du tout. Donc, il y a cette instance qui est élue sur des critères que l'on peut toujours discuter, mais qui est élue. Je trouve tout à fait normal qu'à ce niveau-là toutes les organisations représentatives aient pu participer à l'élection et je serais tout à fait opposé à ce que l'on en fasse des critères doctrinaux pour exclure tel ou tel groupe. Le seul critère serait que ce groupe porte atteinte à l'ordre public. Là, effectivement, il peut y avoir des mesures prises. Tant que ces groupes sont légaux et s'ils se situent dans la légalité, il n'y a aucune raison, ou alors ce serait vraiment tourner le dos à la loi de 1905 qui n'a justement pas voulu favoriser un catholicisme dit républicain face au catholicisme officiel, de favoriser un Islam dit modéré face à un autre Islam qui serait plus orthodoxe...

Maintenant, une fois que ce Conseil est donc fait, il y a deux conditions pour qu'il réussisse : la première condition est que les diverses tendances musulmanes qui le composent travaillent ensemble et ne se déchirent pas par des conflits ou, plutôt, des tensions internes. Qu'il y ait des tensions est normal, logique, mais il ne faut pas que ces tensions dépassent un certain seuil et qu'elles paralysent le fonctionnement de ce Conseil. La seconde condition est que les pouvoirs publics jouent complètement le jeu. C'està-dire qu'ils ont mis en place ce Conseil national du culte musulman et des Conseils régionaux du culte musulman, dans les régions, qui fonctionnent aussi. Maintenant, il ne s'agit plus de prendre à tel ou tel endroit l'imam qui vous plaît ou le musulman de service, etc. Il s'agit vraiment de travailler avec ces instances représentatives, de les prendre au sérieux. Il s'agit aussi de ne pas les mettre en avant à certains moments et en arrière à d'autres. On les a mis en avant au moment de l'enlèvement de deux journalistes en septembre-octobre 2004, et là ces instances ont bien montré leur utilité. J'ai l'impression qu'à d'autres moments, on ne tient pas assez compte d'elles et on veut faire marche arrière. Il ne faut pas faire de marche arrière, il faut jouer le jeu puisqu'on a mis en place une instance.

Si ces deux conditions sont réunies, je pense qu'effectivement dans le moyen terme, cela aidera. Il ne s'agit pas de donner une solution miracle, mais cela aidera à résoudre un certain nombre de problèmes. Si ces deux conditions ne sont pas réunies du tout, alors le remède risque d'être pire que le mal puisque, finalement, avoir une instance qui ne fonctionne pas ce serait encore plus déplorable que de ne pas avoir d'instance.

\section{G.A. : Appartient-t-il à l'État français de former des imams?}

J.B.: Non, pas de former des imams. Que l'État français aide à cette formation, dans la mesure où cette formation demande un dispositif financier et que finalement dans la formation des imams il peut y avoir des enseignements qui correspondent à des données de connaissances objectives... Si vous voulez, dans la formation des imams, il y a des choses qui ont trait uniquement à la confession islamique, à ces convictions. Pour moi, cela doit rester en dehors de l'État. En revanche, si les imams ont besoin de cours de langue, s'ils ont besoin de mieux connaître l'histoire et la culture françaises puisqu'il y a des problèmes d'acculturation effectivement, que l'État aide à cette formation, je trouve cela tout à fait légitime. Je serais d'ailleurs sur la même position pour les autres confessions en séparant bien d'un côté ce qui a trait à la transmission et à la réflexion « convictionnelles » (ce qui est spécifique à la confession et ce qui n'est pas généralisable) et, par contre, de l'autre côté, ce qui a trait à des connaissances générales. Là, pour toutes les confessions, l'État peut mettre en place un dispositif où n'importe qui peut bénéficier de cette aide étatique. Donc voilà, j'aurais une réponse un peu nuancée.

G.A.: Intervention de l'État dans la création du CFCM, aide à la formation des imams... Finalement, sommes-nous encore dans un régime de séparation?

J.B. : Je crois qu'il faut bien distinguer principes et pratiques. C'est-à-dire qu'au niveau des pratiques, c'est vrai, on 
n'est pas complètement dans le régime de séparation pour l'Islam. D'ailleurs, on n'est pas forcement à $100 \%$ dans un régime de séparation pour les autres confessions, mais pour l'Islam le gallicanisme est accentué par rapport à d'autres confessions. Ceci dit, j'aurais une attitude pragmatique en disant: que l'on ait à faire à une religion nouvelle dans l'hexagone, nouvelle dans le sens du poids numérique, qu'il y ait, en partie, à refaire l'histoire et non pas tout de suite être dans le résultat de cette histoire ne m'indigne pas. À condition que l'on sache bien que l'on n'est pas dans un régime de complète séparation et que l'objectif est de parvenir à ce régime de séparation. Et il faudrait se donner un délai raisonnable pour atteindre cet objectif d'amener le rapport entre l'État et l'Islam au même degré de séparation qu'entre l'État et les autres confessions. Mais que ce ne soit pas tout de suite le cas, que l'on ne veuille pas toute de suite être dans l'objectif final, personnellement ne me scandalise pas. Des fois le mieux est l'ennemi du bien.

G.A.: Comme le proposent certains, pensez-vous qu'il faille réviser la loi de 1905 et, dans l'affirmative, sur quels aspects?

J.B.: Non, personnellement, je ne le pense pas. On peut toujours faire tel ou tel aménagement technique. Cela a déjà été fait plusieurs fois. Pourquoi pas une fois de plus... Il y a eu huit aménagements techniques faits depuis 1905, pourquoi pas le neuvième, ou certains disent pourquoi pas le dixième, etc. Quand on parle de réviser la loi de 1905... Je pense d'abord que l'année 2005 est la plus mauvaise année pour réviser la loi de 1905 puisque c’est le principe même de la loi que l'on commémore, ce sont les fondamentaux de la loi que l'on commémore. Sauf pour les gens qui voudraient changer les fondamentaux, mais je ne crois pas, je crois que personne n'a cette idée en tête. Ce n'est pas en 2005 qu'on va faire des aménagements techniques parce que justement il y aurait un court-circuit entre les fondamentaux et les aménagements techniques. Je crois que l'année 2005 doit être une année de célébration raisonnée de la loi et une réflexion autour de l'esprit de la loi, autour de ce qu'elle a apporté, autour de ce qu'elle a perdu, etc. Je pense que dans ses structures fondamentales, il ne faut pas la changer. Qu'elle ne plaise pas à tout le monde est certainement vrai. Mais justement, ce qu'il y a de précieux dans cette loi est qu'elle donne des frustrations équivalentes à des gens très divers. Personne n'est à $100 \%$ satisfait mais personne n'est à $100 \%$ frustré. Pour moi ce sont de grandes lois qui réalisent cet équilibre-là. C'est vraiment une grande loi, une loi emblématique. Ce qu'il ne faut pas faire, parce que cela peut dénaturer l'esprit de la loi, c'est appliquer automatiquement à la loi de 1905 les modifications de la loi de 1901. Donc, mieux séparer la loi de 1905 et la loi de 1901 parce que les modifications de la loi de 1901 sont faites en pensant à la logique propre des associations de loi 1901, elles ne sont pas faites en pensant à logique de la loi de 1905. S'il y a deux lois, chacune doit avoir sa vie autonome. Ensuite, même pour les aménagements techniques, il faut faire attention qu'ils ne détruisent pas l'équilibre de la loi, que cela n'apporte pas de modifications à l'équilibre de la loi. Si vous modifiez l'équilibre de la loi, alors tout le monde va vouloir plus, ce qui est logique. Dans ce cas, on part dans une spirale. Au demeurant, je pense qu'au lieu de perdre son temps et son énergie pour savoir s'il faut ou s'il ne faut pas modifier la loi de 1905, il serait plus intelligent de dire qu'il y a de nouveaux problèmes qui n'existaient pas en 1905 et ce sont ces nouveaux problèmes qu'il faut traiter. Ce sont les problèmes de discrimination indirecte ou directe d'ailleurs, à partir de cette diversité ethnique et culturelle. Quand quelqu'un est discriminé à cause de son prénom ou la couleur de sa peau c'est bien une discrimination ethnique, il faut appeler les choses par leurs noms. Donc, ce sont ces problèmes-là qu'il faut régler. La création d'une autre autorité contre les discriminations peut être un instrument pour aider à régler ces questions. À condition que cela ne soit pas quelque chose qui ne donne que de pseudo-réponses. Il ne faut pas être pessimiste, j'espère beaucoup de cette autre autorité pour élaborer justement des solutions nouvelles et c'est cela qui me semble le plus important. La loi de 1905 a prouvé son efficacité, donc ce n'est pas cela notre problème d'aujourd'hui. Notre problème d'aujourd'hui, c'est finalement de prendre en 2005, par rapport à la situation de 2005, des mesures aussi intelligentes que l'on a prises en 1905 par rapport à la situation d'alors.

G.A.: J'en viens à deux questions concernant l'application territoriale de la laïcité française. Première question, francofrançaise: l'Alsace-Moselle peut-elle rester indéfiniment en dehors du droit laïque?

J.B.: Alors, justement, je ne pense pas qu'elle puisse rester indéfiniment en dehors du droit laïque, et je serais tenté de vous répondre que je serais pour appliquer à l'AlsaceMoselle le même modèle, le même processus que j'ai indiqué pour les imams. C'est-à-dire que l'on se donne du temps pour modifier les choses, mais on a un objectif de modification. Et je regrette que l'on ne l'ait pas eu il y a vingt ans parce que là l'objectif aurait pu être de modifier les choses d'ici 2005. Cela ne peut pas rester indéfiniment cette situation figée. Les autres concordats avec l'Église catholique ont étés renégociés après Vatican II. Là, on ne négocie rien : le Concordat date de 1801 et une de ses conséquences est que le chef d'État français est le dernier chef d'État au monde à nommer les évêques catholiques. Ce n'est pas une situation qui peut durer éternellement. À mon avis, ce n'est pas un hasard si c'est en Alsace qu'il y a eu le plus d'affaires de foulard. Il y a quand même une contradiction entre le fait que le clergé catholique, protestant et juif, soit payé par l'État, qu'il y ait des cours confessionnels de religions à l'école et que l'on interdise en même temps aux jeunes filles de porter un foulard, même discret. Donc, il faut avoir une procédure de concertation avec les gens d'Alsace- Moselle, il faut aussi se demander si des choses qui sont actuellement rétrogrades en AlsaceMoselle ne pourraient pas être modifiées pour être, au 
contraire, un laboratoire expérimental d'innovation. Par exemple: si on changeait les cours confessionnels de religions en enseignement laïque sur la religion, on ferait passer l'Alsace-Moselle de l'arrière à l'avant-garde et on pourrait expérimenter cet enseignement laïque des religions. Il y a des heures de cours qui sont prévues, qui existent déjà. Donc le dispositif formel est déjà en place. Du coup cela donnerait un peu de corps à cette idée d'enseignement laïque des religions. Quand je vais dans d'autres pays européens ou même au Japon, le gens me disent : «Mais enfin, pourquoi la France n'est-elle pas plus audacieuse, elle émet cette idée d'enseignement laïque de religions et elle ne passe pas à l'acte. Cela nous intéresserait beaucoup si elle passait à l'acte. » C'est intéressant car il y a en Europe des cours confessionnels de religions et ce serait se substituer à ces cours confessionnels de religion. Au Japon il y a interdiction, dans la Constitution, de l'enseignement confessionnel de la religion. C'est une constitution très laïque, plus laïque même que la Constitution française par certains côtés, mais ils ont aussi ce besoin d'un enseignement laïque des religions et ils disent que cela est bien dommage que la France ne passe pas à l'acte. Là, il y aurait une possibilité de profiter de la situation un peu spéciale d'Alsace-Moselle pour la retourner en expérimentation laïque.

G.A.: Seconde question territoriale: quel est, selon vous, l'avenir de cette exception française que constitue la laïcité en Europe? S'agit-il d'un modèle transposable?

J.B.: Je ne l'entendrais pas comme une exception française parce que si je décompose les trois éléments que je vous ai donnés, ces trois éléments existent dans d'autres pays ou, en tout cas, correspondent à des débats qui existent dans d'autres pays. Ce que je constate, c'est que cette expression «laïcité exception française » date de 1990, je ne la rencontre pas avant. Donc, la laïcité française a vécu des décennies et des décennies sans que l'on parle d' « exception française». Je vous l'ai dit, la Constitution du Japon est tout à fait laïque, celle du Mexique aussi. Même dans des pays où le terme de laïcité n'est pas employé, vous vous apercevez qu'il y a des situations analogues à la situation française... voire même plus laïques. Par exemple, au Japon il y a interdiction que l'argent public aille à des organismes confessionnels. Ce qui n'est pas le cas en France, notamment avec la loi Debré.

Je dirais qu'en France pas plus que dans d'autres pays, il n'y a de la laïcité absolue. C'est tant mieux parce que lorsqu'on veut réaliser l'absolu, politiquement, on risque de le faire au détriment de la liberté et de la démocratie. Ce que je souhaite, c'est quand même qu'il y ait un certain nombre de points de la laïcité qui soient présentés de manière dynamique en Europe. Je pense notamment à la séparation de l'Église et de l'État, le fait qu'il n'y ait pas de religion officielle ou semi-officielle. Je pense que c'est quelque chose que l'on pourrait plus facilement exporter. En même temps, on se rend compte que suivant le contexte, suivant les traditions nationales, suivant les pays, cette laïcité se concrétise de manière un peu différente. Effectivement, il y a peut-être une difficulté à exporter le mot même de «laïcité » parce qu'on l'applique trop à la manière dont la laïcité est vécue en France. Mais, justement, je travaille, entre autres, avec des collègues mexicains qui parlent de la laïcité à propos du Mexique, des collègues canadiens qui parlent de la laïcité québécoise, etc. Le terme commence à être assez couramment utilisé, en tout cas dans le milieu de gens qui font des analyses. Donc, il n'y a pas à être timide à ce niveau-là mais à condition de bien voir que l'on est dans l'import-export. C'est-à-dire que notre attitude sera considérée comme arrogante par les autres si l'on dit que l'on veut exporter notre modèle et c'est tout. Il faut le faire plus dans une optique d'échange. J'ai vous ai donné un exemple avec le Québec: il y a, effectivement, certains aspects de la laïcité française qui sont tout à fait exportables au Québec et, inversement, la notion d'accommodement raisonnable peut être importée et peut donner des choses intéressantes en France. Donc, c'est dans ce mouvement un peu de dialogue, d'échange, de circulation que je vois la laïcité française, et je pense qu'elle peut répondre aux défis du $\mathrm{XxI}^{\mathrm{e}}$ siècle.

G.A.: Pensez-vous que le traité établissant une Constitution pour l'Europe menaçait véritablement la lä̈cité française?

J.B.: Non, il y a eu des contrevérités qui ont été dites. Par exemple, on a parlé de l'article de la charte où il était question de la possibilité de manifester sa religion et ses convictions. D'abord «sa religion et ses convictions » : il n'est pas question que de la religion mais aussi des convictions philosophiques non religieuses. Ensuite, on a fait comme si cet article de la charte était une nouveauté. Or, vous savez que c'est une reprise, exactement, de la Convention européenne des droits de l'homme de 1950 à laquelle la France a adhéré; de plus, elle a accepté la juridiction de la Cour européenne des droits de l'homme. Donc, la France accepte d'être jugée selon cet article déjà depuis 30 ans. Ainsi, présenter cela comme quelque chose de nouveau qui menacerait la laïcité française, c'est une double erreur. Une erreur d'abord historique, et ensuite cela fonctionne depuis 30 ans et depuis 30 ans cela n'a absolument pas menacé la laïcité française. Cela fait partie des récupérations un peu politiciennes dont je vous ai parlé tout à l'heure: il y a des gens qui prennent des libertés avec la véracité des choses soit pour des raisons politiques, soit pour des conceptions de la laïcité qui sont très réductrices et qui ne fonctionnent pas. Dans le cas où la France ne respecterait pas la liberté de manifestation des convictions religieuses, il s'agit de manifestations qui ne doivent pas déroger à l'ordre public, c'est clair et c'est aussi prévu par l'article 9 de la Convention des droits de l'homme, il pourrait y avoir un recours devant la Cour européenne des droits de l'homme et la France pourrait être tout à fait condamnée. Donc, on a créé des problèmes là où il n'y en avait pas. Le seul problème qu'il y avait c'était l'article 52, troisième alinéa, qui parle du dialogue régulier avec les religions et les convictions. Là, tout dépend de ce qu'on 
appelle « dialogue». C'est vrai que si on veut dire qu'il y aurait un retour de certaines normes religieuses qui surplomberaient la société globale, dans ce cas ce serait dangereux. S'il s'agit d'un dialogue: on discute, on s'informe, on voit les avis des uns et des autres, là encore religions et convictions, les convictions philosophiques étant mises sur le même plan que les religions, il est normal que l'on prenne l'avis de tout le monde, que l'on s'informe de l'avis de tout le monde. Tout dépend de ce que l'on entend par le terme de dialogue. Cela ne veut pas dire qu'il n'y ait pas de vigilance à avoir. Mais cette vigilance ne peut pas être crispée, ne peut pas donner d'impression de forteresse assiégée. Elle fait partie de la vie. Dans la vie, il faut toujours avoir un certain degré de vigilance. Le dialogue existe déjà en France. Il a été instauré de manière plus régulière par Lionel Jospin lui-même. Et personne n'a dit qu'il y a eu une atteinte frontale à la laïcité. À partir du moment où l'État et le pouvoir public restent maîtres de leurs décisions et qu'il s'agit uniquement de consultation, tous les éléments de la société civile peuvent être consultés sans que cela ne déroge au principe de la laïcité. Ce qu'il ne faudrait pas, c'est redonner aux groupements religieux, ou « convictionnels ", la possibilité de surplomber la société globale. Mais ce n'est pas le cas de l'évolution en Europe. Même si, évidemment, la sortie du régime communiste a changé un peu les choses pour certains pays, la plupart des pays européens adoptent plutôt une évolution inverse. Regardez l'exemple de l'Espagne qui est tout à fait probant. En Espagne, on est passé d'une structure très cléricale sous Franco à une structure beaucoup plus libérale. On peut dire qu'en Italie, à moindre degré, aussi. L'évolution d'État démocratique est une évolution quand même sécularisatrice voire même laïcisatrice dans beaucoup de pays si l'on voit ce qui s'est passé ces dernières décennies. Donc, je pense que là, il y a eu des crispations qui n'ont pas été politiquement innocentes.

G.A.: Donc, en conclusion, si l'on devait poser la question: "quel avenir pour la laïcité française?", pourrait-on répondre que le $\mathrm{XXI}^{e}$ siècle sera lä̈que?

J.B. : Il sera laïque si la laïcité sait répondre aux défis du XxI ${ }^{e}$ siècle. Pour donner un exemple : au niveau de la médecine, le grand mot d'ordre du $\mathrm{xx}^{\mathrm{e}}$ siècle était l' "espérance de vie », le mot d'ordre pour le $\mathrm{xxI}^{\mathrm{e}}$ siècle il semble que cela va être «mourir dans la dignité ». Et «mourir dans la dignité » repose la question anthropologique : qu'est-ce que l'homme? Qu'est-ce que l'être humain?, etc. Quand il s'agissait de prolonger l'espérance de vie et que la vie était encore plus ou moins considérée comme un phénomène naturel par tout le monde, comme c'était encore le cas il y a 50 ans, tout le monde pouvait dire : on a dans le privé des conceptions différentes, ces conceptions restent dans le privé et on se bat tous pour le progrès médical prolongeant l'espérance de vie. Â partir du moment où le mot d'ordre devient «mourir dans la dignité », on se retrouve dans l'euphémisation de la frontière du public et du privé parce que cela veut dire que tout est lié à des projets de vie. Ainsi, il est normal que les options je dirais symboliques, spirituelles, au sens large, de chacun soient prises en compte. Car les gens n'auront pas forcement le même rapport à la mort et la même conception de la manière de mourir dans la dignité. La laïcité doit tenir compte de cela sans qu'une interprétation devienne hégémonique, obligatoire et s'impose face aux autres. Donc, il y aura toujours à résister à des cléricalismes ou à des néo-cléricalismes. Cela fait partie de la vigilance dont j'ai parlé.

En même temps, si la laïcité n'est pas en mouvement, si elle ne bouge pas et si elle reste sur des positions qui correspondaient à des problèmes d'une certaine époque et qu'elle ne prend pas en compte que maintenant il y a d'autres problèmes, elle deviendra elle-même quelque chose de dogmatique, de figé, de passéiste et, à ce moment-là, elle ne saura plus répondre aux défis du siècle. À ce moment-là elle deviendra une nostalgie. Ainsi, la réponse est dans la vitalité de tous ceux qui se rattachent activement à la laïcité. 